\title{
Most Common Isolates in Neonatal Sepsis, a Prospective Study in Tertiary Care Hospital
}

\author{
Patta Apparao*, Jyoti Nagdev and P. Siddartha \\ ${ }^{1}$ Department of Microbiology, Late Shri Lakhiram Agrawal Memorial \\ Medical College, (C.G.), India \\ ${ }^{2}$ Dr. C.V.R. University, Kota, Bilaspur (C.G.), India \\ ${ }^{3}$ NRI, Medical College, Vishakhapatnam, India \\ *Corresponding author
}

\section{A B S T R A C T}

Neonatal sepsis is one of the most common causes of mortality and morbidity, particularly the developing countries. Accurate and timely diagnosis of neonatal sepsis remains a major challenge to the pediatricians and neonatologists. The objective of this study was to determine the causative bacteria and pattern of susceptibility to antibiotics in NICU of a

\section{Keywords}

Neonates,

Empirical therapy, Early onset sepsis, late onset sepsis, Antimicrobial sensitivity.

\section{Article Info}

Accepted:

17 July 2017

Available Online:

10 September 2017 tertiary care center, which in turn may help in implementation of empirical therapy. This prospective study was carried out in the department of microbiology, Govt. medical college Raigarh (c.g.) during Jun 2016- Jun 2017. A total of 255 cases of blood cultures are donein our microbiology department. Out of which, 120 cases were positive (Bac Talert) for culture. A total of 120 cultures were found to be positive out of 255 cases (47.0\%). The most common organism isolated was Staphylococcus aureus (40.2\%), followed by Klebsiella pneumonia (37 \%) and Escherichia coli (14\%) other organisms were much less in number. which included pathogenic Streptococci, coagulase negative Staphylococcus (CoNS), Pseudomonas, Acinetobacter and Enterobacter species. Candida albicans also isolated. The gram positive organisms except Streptococci displayed a high degree of resistance to most penicillins and ciprofloxacin but were sensitive to vancomycin, amikacin and linezolid. There was a high incidence of resistance noted with ampicillin, gentamicin and ciprofloxacin, amongst most gram negative organisms' where in ceforuxime, amikacin and meropenem, imipenem, netilmycin were effective in most cases. There is an increasing trend of antibiotic resistance to the commonly used first line drugs. Continuous surveillance for antibiotic susceptibility is needed to ensure proper empirical therapy.

\section{Introduction}

Sepsis is one of the commonest causes of neonatal morbidity and mortality. It is responsible for about $30-50 \%$ of the total neonatal deaths in developing countries $(1,2)$. Neonatal infections are estimated to cause 1.6 million deaths every year globally, and $40 \%$ of all neonatal deaths occur in developing countries $(3,4)$. According to diagnosis of national neonatal sepsis database (NNPD) 2000 neonatal sepsis is the most common cause of deaths in the country followed by prematurity and birth asphyxia $(5,6)$.

Neonatal sepsis is of two types; early onset sepsis and late onset sepsis. Early onset sepsis (EOS) present within first 72 hours of life. In 
severe cases, the neonates may be symptomatic at birth. Infants with EOS usually present with respiratory distress and pneumonia. The source of infection is generally the maternal genital tract (7). Late onset sepsis usually presents after 72 hours of age. The source of infection is either nosocomial or community acquired, and neonates usually presented with septicemia, pneumonia or meningitis (8). Early diagnosis and proper management can reduce the neonatal mortality but aetiological agent do not remain the same and include a wide variety of both gram positive and gram negative bacteria. One should know the usual aetiologic agent and its antibiotic susceptibility patterns in community, before commencing empirical therapy.

\section{Materials and Methods}

A Prospective study was conducted on the blood samples sent from Kirodimal government hospital neonatal intensive care unit, in the Department of Microbiology, Late Shri Lakhiram Agrawal Memorial Medical college Raigarh (C.G.). India, with due permission of the ethical committee of our institute. In this analysis, we analyzed the data of culture and sensitivity pattern of cases of neonatal sepsis from Jun 2016-Jun 2017.

Blood for bacterial culture was collected aseptically and $2 \mathrm{ml}$ of blood was added to each of two bottles containing $20 \mathrm{ml}$ of Tryptone soya broth (Hi-Media Labs. Mumbai, India) the bottles were incubated aerobically at $37^{\circ} \mathrm{C}$ for 7 days and sub cultured on sheep blood agar and MacConkey agar overnight, for $48 \mathrm{~h}$ or for 7 days or for an in-between period when visible turbidity appeared. In positive cases, Gram - positive isolates were identified at the species level by conventional biochemical and serological tests (9). In babies in whom coagulase negative staphylococcus aureus (CONS) was isolated in the first 3 days of life, a repeat blood culture was performed to confirm the infection. Proven sepsis was defined as the presence of clinical features of sepsis along with the isolation of an organism in the blood culture. All organisms were classified based on the time-point at which the blood was collected for culture as follows; those causing early onset sepsis (EOS-less than and up to 72 $\mathrm{h}$ of life) and those causing late onset sepsis (LOS-greater than $72 \mathrm{~h}$ of life). The organisms were further classified based on their Gram's stain status.

\section{Results and Discussion}

A total of 120 cultures were found to be positive out of 255 cases $(47.0 \%)$. The most common organism isolated was Staphylococcus aureus (40.3\%), followed by Klebsiella pneumoniae (37 \%) and Escherichia coli (14\%) other organisms were much less in number; which included pathogenic Streptococci, Coagulase negative Staphylococcus (CoNS), Pseudomonas, Acinetobacter and Enterobacter species. Candida albicans also isolated. A total 255 neonates were included in the present study, $120(47.0 \%)$, yielded positive cultures (Table $1)$.

All isolates showed low sensitivity to ampicillin, ciprofloxacin and gentamicin, good sensitivity to cefotaxime, and maximum sensitivity to amikacin, cefepime meropenem, vancomycin, linezolid and clindamycin. Staphylococcus aureus was absolutely resistant to penicillin but showed $100 \%$ sensitivity to vancomycin.

In our study all the isolates were resistant to penicillin. Ampicillin, gentamicin and ciprofloxacin shown lowest sensitivity with highest sensitivity to either cefotaxime or amikacin and hence a co-prescription of these two antibiotics appear prudent as the initial 
choice while awaiting for the blood culture reports (Table 2). This combination has given us the best results in our neonatal intensive care unit. In developing countries, rates of blood stream infections (BSI) have been reported to be 1.7 to 33 per 1,000 live births, with rates in Asia clustering around 15 per 1,000 live births. (10). Blood culture has remained the gold standard for the confirmation of sepsis (11). The rate of admission of early and late onset sepsis as well as the prevalence of organisms and their sensitivity patterns were much similar (12). This may be due to the fact that not only the vertical transmission but also the horizontal spread of infection may play a part in the early onset of sepsis in hospitalized neonates $(13,14)$.

Table.1 Bacterial isolates causing neonatal sepsis $(\mathrm{n}=120)$

\begin{tabular}{|l|l|c|}
\hline S. No. & Bacterial isolates & $\%$ of isolation \\
\hline 1. & Staphylococcus aureus & $(40.3 \%)$ \\
\hline 2. & Klebsiella pneumonia & $(37 \%)$ \\
\hline 3. & Escherichia coli & $(14 \%)$ \\
\hline 4. & Streptococcus spp. & $(4.4 \%)$ \\
\hline 5. & Coagulase negative staphylococcus & $(2.1 \%)$ \\
\hline 6. & Psudomonas spp & $(1.2 \%)$ \\
\hline 7. & Acinetobacter spp & $(1.0 \%)$ \\
\hline
\end{tabular}

Table.2 Antimicrobial sensitivity pattern of gram negative bacteria

\begin{tabular}{|l|l|c|c|}
\hline S. No. & Antibiotics & Concentration & \% of Sensitivity \\
\hline 1. & Amikacin & $30(\mu \mathrm{g})$ & $24.4 \%$ \\
\hline 2. & Amoxiclave/clavulanic acid & $30(\mu \mathrm{g})$ & $10.5 \%$ \\
\hline 3. & Ampicillin & $10(\mu \mathrm{g})$ & $8.8 \%$ \\
\hline 4. & Cefepime & $30(\mu \mathrm{g})$ & $20.5 \%$ \\
\hline 5. & Ceftazidime & $30(\mu \mathrm{g})$ & $11.1 \%$ \\
\hline 5. & Ceftriaxone & $30(\mu \mathrm{g})$ & $11.1 \%$ \\
\hline 6. & Ceferuxime & $30(\mu \mathrm{g})$ & $20.0 \%$ \\
\hline 7. & Ciprofloxacin & $5(\mu \mathrm{g})$ & $10.2 \%$ \\
\hline 8. & Gentamicin & $10(\mu \mathrm{g})$ & $10.0 \%$ \\
\hline 9. & Imipenem & $10(\mu \mathrm{g})$ & $75 \%$ \\
\hline 10. & Meropenem & $10(\mu \mathrm{g})$ & $71.0 \%$ \\
\hline 11. & Netilmicin & $30(\mu \mathrm{g})$ & $13.3 \%$ \\
\hline 12. & Piperacillin/Tazobactam & $100 / 10(\mathrm{~g})$ & $17.7 \%$ \\
\hline 13. & Tobramycin & $10(\mu \mathrm{g})$ & $11.1 \%$ \\
\hline 14 & Piperacillin & $100(\mu \mathrm{g})$ & $15.5 \%$ \\
\hline 15 & Colistin & $10(\mu \mathrm{g})$ & $60.5 \%$ \\
\hline 16 & Cefoperazone & $75(\mu \mathrm{g})$ & $19.5 \%$ \\
\hline 17. & Cefotaxime & & $21.4 \%$ \\
\hline
\end{tabular}


Table.3 Antimicrobial sensitivity pattern of gram positive bacteria

\begin{tabular}{|l|l|l|l|}
\hline S. No. & Antibiotics & Concentration & $\%$ of Sensitivity \\
\hline 1. & Amikacin & $30(\mu \mathrm{g})$ & $24.4 \%$ \\
\hline 2. & Amoxiclave /clavulanic acid & $30(\mu \mathrm{g})$ & $10.0 \%$ \\
\hline 3. & Ampicillin & $10(\mu \mathrm{g})$ & $7.5 \%$ \\
\hline 4. & Cefepime & $30(\mu \mathrm{g})$ & $13.3 \%$ \\
\hline 5. & Ceftazidime & $30(\mu \mathrm{g})$ & $11.1 \%$ \\
\hline 5. & Ceftriaxone & $30(\mu \mathrm{g})$ & $15.2 \%$ \\
\hline 6. & Cefuroxime & $30(\mu \mathrm{g})$ & $18.0 \%$ \\
\hline 7. & Ciprofloxacin & $5(\mu \mathrm{g})$ & $10.2 \%$ \\
\hline 8. & Gentamycin & $10(\mu \mathrm{g})$ & $10.2 \%$ \\
\hline 9. & Imipenem & $10(\mu \mathrm{g})$ & $78 \%$ \\
\hline 10. & Meropenem & $10(\mu \mathrm{g})$ & $75 \%$ \\
\hline 11. & Netilmicin & $30(\mu \mathrm{g})$ & $13.3 \%$ \\
\hline 12. & Piperacillin/Tazobactam & $100 / 10(\mathrm{~g})$ & $17.7 \%$ \\
\hline 13. & Tobramycin & $10(\mu \mathrm{g})$ & $11.1 \%$ \\
\hline 14 & Linezolid & $30(\mu \mathrm{g})$ & $71.1 \%$ \\
\hline 15 & Oxacillin & $1(\mu \mathrm{g})$ & $14.2 \%$ \\
\hline 16 & cefotaxime & $30(\mu \mathrm{g})$ & $21.0 \%$ \\
\hline 17 & Vancomycin & $30(\mu \mathrm{g})$ & $100 \%$ \\
\hline 18 & Clindamycin & $5(\mu \mathrm{g})$ & $19.0 \%$ \\
\hline
\end{tabular}

The most common organism identified in our study was Staphylococcus aureus (40.3\%). The other gram positive organisms to be isolated were Streptococcal species and coagulase negative Staphylococcus. Most of the studies have found a preponderance of gram negative organisms like Klebsiella, Pseudomonas and Enterobacter species. However Staphylococcus $(40.0 \%)$ was the commonest gram positive to be isolated in most of the studies (Table 3).

Klebsiella pneumoniae was the most common gram negative organism (37\%) and the second most frequent after staphylococcus aureus in the study. This finding is not in accordance with NNPD 2002-03 data, where the most common organisms causing neonatal sepsis was Klebsiella pneumoniae followed by staphylococcus aureus, pseudomonas. In our study, all the isolates were resistant to penicillin, ampicillin. gentamicin and ciprofloxacin had lowest sensitivity to all bacterial isolates, Highest sensitivity was recorded with imipenem, meropenem and vancomycin followed by amikacin and cefepime. vancomycin showed sensitivity of $100 \%$. As far as cephalosporins are concerned, moderate sensitivity was observed for third generation cephalosporins i.e., cefotaxime while higher sensitivity was documented for fourth generation cephalosporins i.e., cefepime, Low sensitivity of commonly used antibiotics and fair sensitivity to amikacin was also observed by other authors.

Neonatal sepsis is a leading cause of neonatal admission, morbidity and mortality in developing countries. Bacterial spectrum could be different in different regions. Sensitivity pattern also differs accordingly. The antibiotics susceptibility pattern in our study suggested. Initial empirical choice is of cefotaxime in combination with amikacin. A low susceptibility to commonly used 
antibiotics like ampicillin and gentamicin is a cause for concern. The knowledge of prevailing strains and the antibiotic sensitivity patterns in the region is mandatory for each center due to temporal changes in the causative organisms and their antibiotic susceptibility. Periodic evaluations not only reveals the recent trend of increasing resistance to commonly used antibiotic but also helps in implementation of a rational empirical therapy.

\section{References}

1. Bang AT, Bang RA, Bactule SB, Reddy HM, Deshmukh MD, Effect of home based neonatal care and management of sepsis on neonatal mortality : field trial in rural India .Lancet, 1999;354 : 1955-61.

2. Stoll BJ. The global impact of neonatal infection. ClinPerinatol .1997; 24:1-21.

3. World Health Organization (1993); Motherbaby package; a road map for implementation in countries .World Health Organization, Geneva .

4. Saving Newborn Lives (2001); The state of the world's newborns ; a report from Saving Newborn Lives .Save the Children, Washington .DC.

5. National Neonatologist Forum. Report of National Neonatal Perinatal Database 2000,New Delhi,Available at : http;//www, newbornwhocc,org/pdf/nnpd2002-03,PDF.

6. Meherban S. Perinatal Infection. In; Care of Newborn, Sagar Publication, New Delhi, 2010;7;223-33.

7. Singh M,Narang A, Bhakoo $\mathrm{O} N$, Predictive perinatal score in the diagnosis of neonatal sepsis, J Trop Paediatr, 1994 Dec;40 (6);365-81.

8. Report of the National Neonatal Perinatal Database (National Neonatology Forum) 2002-03.

9. Lipuma, J. J,Currie,B, J, Lum,G,et al. (2007); Burkholderia, Stenotrophomonas, ralstonia, Cupriavidus, Pandoraea, Brevundimonas, Comamonas, Delttia and Acidovorax, p.749-769.In Murrey, P,R, et al (ed), Manualof Clinical Microbiology, American Society for Microbiology, ASM Press, Washington DC

10. Zaidi, A,K,M., Huskins, W,C, Thaver, D., et al, (2005) ; Hospital-acquired neonatal infections in developing countries, Lancet, 365,1175-1188. A report from 18 centers across India (National Neonatal Perinatal Database (NNPD) for 2002-2003 reported an incidence of 8.5 per 1,000 live births for blood-culture -proven sepsis.

11. National Neonatal Perinatal Database (NNPD) 2005; Report for the Year 200203 National Neonatology Forum, India. Blood culture has remained the gold standard for the confirmation of sepsis.

12. Klein JO, Remington JS, Infectious Diseases of the Fetus and the Newborn infant . 5TH ed. Philadelphia ; WB Saunderss;2001,Current concepts of infections of the fetus and the newborn infant, In ; Remin .

13. Viwanathan R, Singh AK, Mukherjee S, Mukherjee R,DasP,BasuS,Aetiology and antimicrobial resistance of neonatal sepsis at a tertiary care centre in eastern India; a 3 year study, Indian J Paediatr, 2011;78;40912.

14. Viswanathan R, Singh AK, Ghosh C, Basu $\mathrm{S}$ Stenotrophomonasmaltophila as a cause of early onset neonatal sepsis, Indian Paediatr,2011;48;397-9.

\section{How to cite this article:}

Patta Apparao, Jyoti Nagdev and Siddartha, P. 2017. Most Common Isolates in Neonatal Sepsis, a Prospective Study in Tertiary Care Hospital. Int.J.Curr.Microbiol.App.Sci. 6(9): 976980. doi: https://doi.org/10.20546/ijcmas.2017.609.118 